

\title{
PMF: The front end electronic of the ALFA detector
}

P. Barrillon, S. Blin, C. Cheikali, D. Cuisy, M. Gaspard, D. Fournier, M.

Heller, W. Iwanski, B. Lavigne, C. de La Taille, et al.

\section{To cite this version:}

P. Barrillon, S. Blin, C. Cheikali, D. Cuisy, M. Gaspard, et al.. PMF: The front end electronic of the ALFA detector. IEEE NSS-MIC 2008 Nuclear Science Symposium - Medical Imaging Conference 16th International Workshop on Room-Temperature Semiconductor X-ray and Gamma-ray Detectors, Oct 2008, Dresden, Germany. pp.1874-1877, 10.1109/NSSMIC.2008.4774756 . in2p3-00450926

\section{HAL Id: in2p3-00450926 https://hal.in2p3.fr/in2p3-00450926}

Submitted on 28 Jan 2010

HAL is a multi-disciplinary open access archive for the deposit and dissemination of scientific research documents, whether they are published or not. The documents may come from teaching and research institutions in France or abroad, or from public or private research centers.
L'archive ouverte pluridisciplinaire HAL, est destinée au dépôt et à la diffusion de documents scientifiques de niveau recherche, publiés ou non, émanant des établissements d'enseignement et de recherche français ou étrangers, des laboratoires publics ou privés. 


\title{
PMF: the front end electronic of the ALFA detector
}

\author{
P. Barrillon ${ }^{\text {a }}$, S. Blin ${ }^{\text {a }}$, C. Cheikali ${ }^{\text {a }}$, D. Cuisy ${ }^{\text {a }}$, M. Gaspard ${ }^{\text {a }}$, D. Fournier ${ }^{\text {a }}$, M. Heller ${ }^{\text {a }}$, W. Iwanski ${ }^{\text {b }}$, B. \\ Lavigne $^{\text {a }}$, C. De la Taille ${ }^{a}$, P. Puzo ${ }^{\text {a }}$ J-L. Socha ${ }^{\text {a }}$ \\ ${ }^{a}$ Laboratoire de l'Accélérateur Linéaire, 91898 Orsay, France \\ ${ }^{\mathrm{b}}$ Institute of Nuclear Physics PAN, Radzikowskiego 152, 31-342 Cracow, Poland (based at CERN, Geneva, Switzerland)
}

\begin{abstract}
The front end electronic (PMF) of the future ATLAS luminometer is described here. It is composed by a MAPMT and a compact stack of three PCBs which deliver the high voltage, route and readout the output signals. The third board contains a FPGA and MAROC, a 64 channels ASIC which can correct the non uniformity of the MAPMT channels gain thanks to a variable gain preamplifier. Its main role is to shape and discriminate the input signals at $1 / 3$ photo-electron and produce 64 trigger outputs. Laboratory tests performed on prototype and pre-series PMFs have showed performances in good agreement with the requirements.
\end{abstract}

\section{INTRODUCTION}

The PMF (section B) is the front end electronics designed for the ATLAS luminometer (section A). It consists of a 64 channels photomultiplier (MAPMT) and a very compact stack of three different PCBs, mounted directly on the back and in the shadow of the MAPMT. The ASIC MAROC (section C) is used for the signal readout and treatment.

\section{A. ALFA detector}

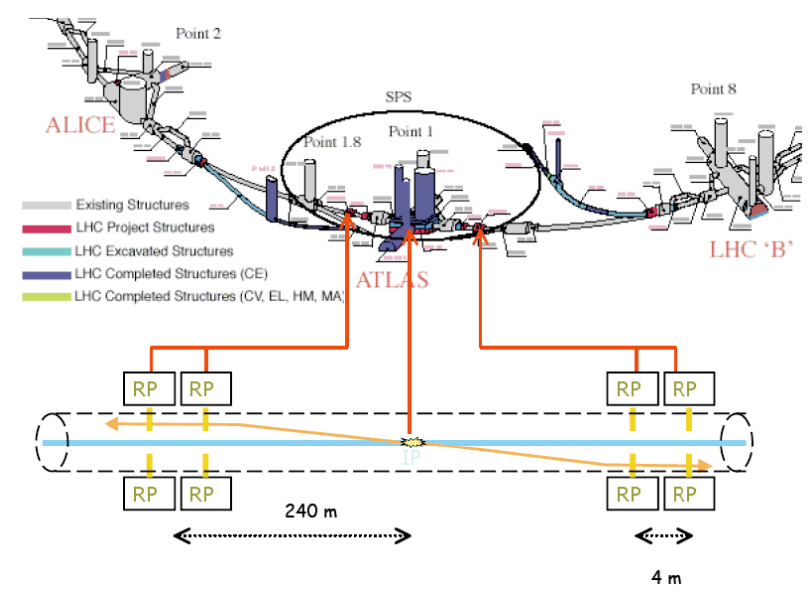

Fig. 1. Schematic view of the Roman Pots location with respect to the LHC beam.

ALFA stands for Absolute Luminosity For ATLAS [1]. This detector is located at $240 \mathrm{~m}$ from the interaction point (Fig. 1) and is meant to detect high energy diffused protons from the LHC beam. It is made of 20 staggered U-V scintillating fiber layers (Fig. 2) inserted in Roman Pots (eight in total). Each of these plans is made of 64 fibers which produce light when a particle comes through them. The light is then collected by multi-anode photomultiplier tubes H7546 from Hamamatsu [2], that will run at 800 to $950 \mathrm{~V}$ which corresponds to a gain of $3.10^{5}$ to $2.10^{6}$. These PMTs have an important non-uniformity that can not be corrected by applying a different high voltage to each channel. Therefore one has to amplify the output signal with different factors for each channel.

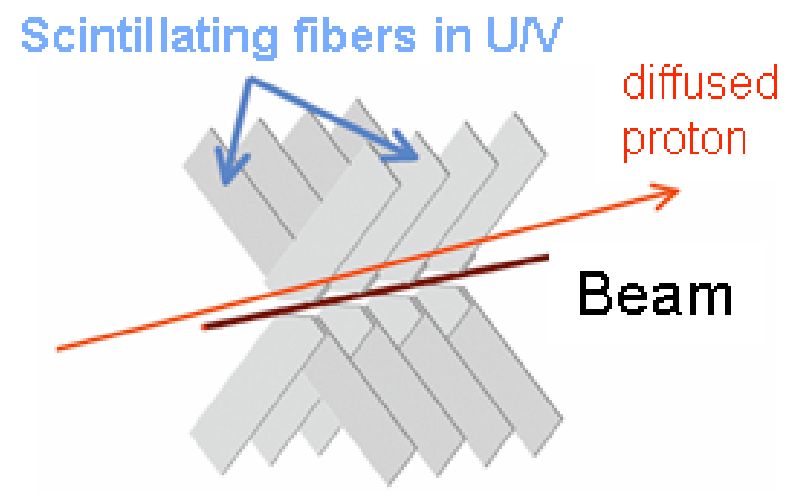

Fig. 2. Schematic view of location of the scintillating fibers with respect to the beam. The U/V layers are arranged orthogonally to each other.

Figure 3 shows a schematic of a Roman Pot and the front end electronic. The whole readout system of ATLAS luminometer is presented in reference [3].

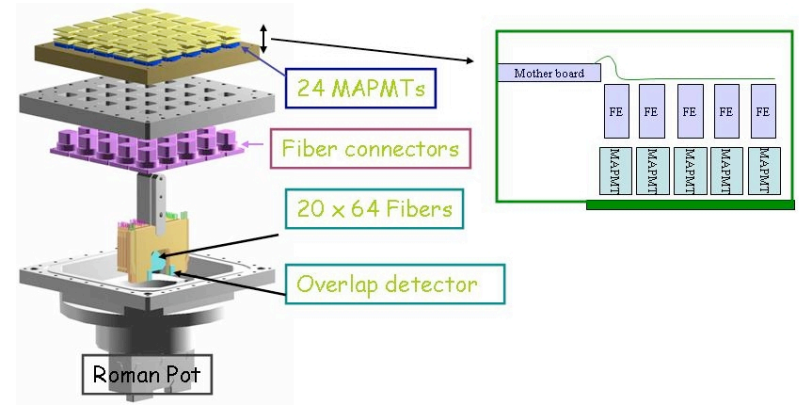

Fig. 3. Exploded schematic view of a Roman Pot and its front end electronic.

\section{B. $\quad P M F$}

The Photomultiplier Front end (PMF) electronic is represented on the Fig. 4. It is made of a MAPMT and three boards $(3 \mathrm{~cm} \times 3 \mathrm{~cm})$ in its shadow:

- The HV board which brings the high voltage to the MAPMT;

- The passive (or intermediate) board which routes the signals to connectors located on the edge;

- The active board which has the read out chip MAROC directly wire-bonded on the PCB on one side and a FPGA (Lattice) on the other side. 

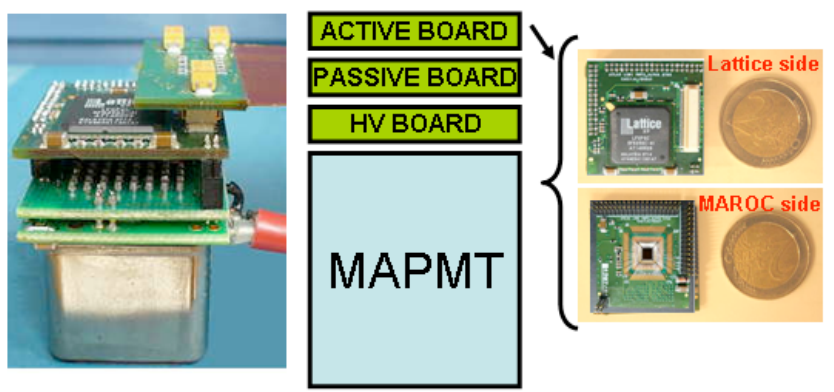

Fig. 4. PMF schematic and picture.

The PMFs will be arranged in a 5 by 5 matrix for each Roman Pot. Each line of up to 5 PMFs will be linked to the mother board through a kapton cable. In total 23 PMFs per Roman Pot will be installed, 20 for the standard scintillating fibers layers and 3 for another detector (overlap detector) described in [1].

The active board is technologically challenging since it consists of 10 different layers, has MAROC located on the sixth layer through a hole in the upper layers and needs different types of vias (see Fig. 5). The bonding wires $(<1 \mathrm{~cm}$ long) are split between the first two layers.

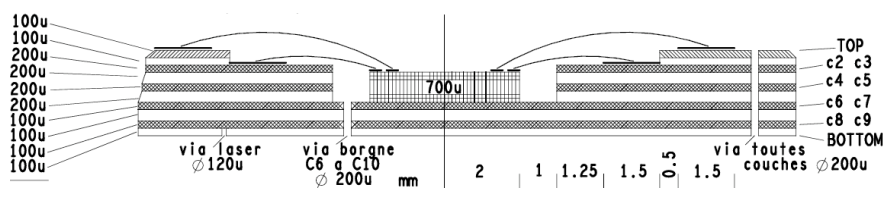

Fig. 5. Schematic view of the active board layer structure.

\section{MAROC}

MAROC (Multi Anode ReadOut Chip) is a 64 inputs ASIC which allows correcting for the gain spread of MAPMT channels thanks to a 6 bits variable gain preamplifier. For each channel the signal is shaped (fast shaper, $15 \mathrm{~ns}$ ) and discriminated to produce a trigger output. The discriminator thresholds are set by an internal 12 bit DAC, made of a 4 bit thermometer DAC for coarse tuning and a 8 bit mirror for fine tuning [4].

A multiplexed charge output is also produced both in analog and digital thanks to a Wilkinson ADC. The block diagram represented on figure 6 summarizes the different features of this chip [4].

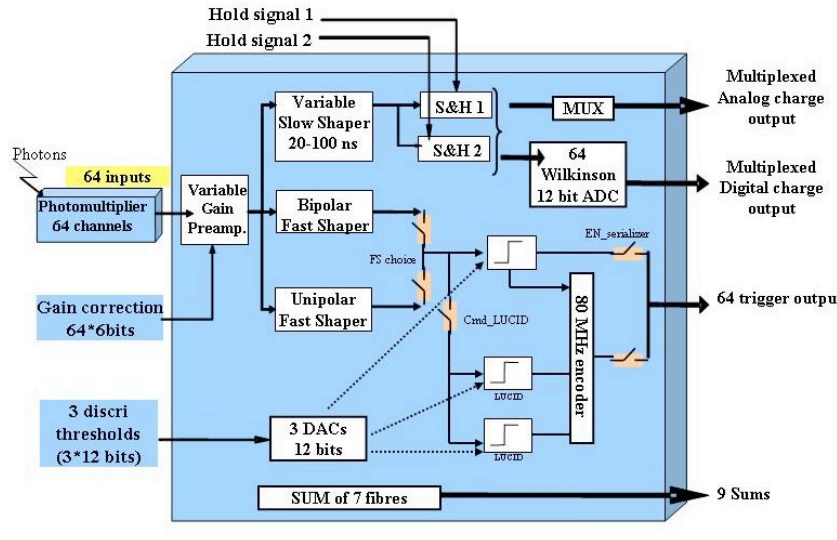

Fig. 6. Block diagram of MAROC second version.

The main requirements are the following: $100 \%$ trigger efficiency for a signal greater than $1 / 3$ of a photoelectron, a charge measurement up to 30 photoelectrons with linearity of $2 \%$ or better and a low cross talk. The performances of the second version of MAROC were checked successfully during the year 2007 at LAL-Orsay [5]. A nice dispersion of the trigger output $( \pm 5 \mathrm{fC})$ was, in particular, observed.

\section{LABORATORY TESTS}

\section{A. Description}

Lots of PMTs and PCBs were tested separately respectively at DESY and LAL-Orsay.

As far as what concerns the PCBs, the critical one to test is the active board due to its complexity and the many steps in its production. It is tested coupled with a passive board. A capacitor is used to inject signal in a single channel. A dedicated test board (left part of figure 7) has been designed for this purpose. Its role is to communicate with up to 5 PMFs via a kapton cable (central part of figure 7) sending configuration and collecting output signals. Standalone C and Labview programs were developed in order to enable testing.

A first batch of 8 active boards, considered as the prototype, was produced during autumn 2007. Thorough laboratory tests were performed at LAL after a period dedicated to the development of both test board (Xilinx) and PMF (Lattice) FPGA firmwares.

One of this active board was used to build a full PMF (3 PCBs and a PMT) in order to carry out a test with a LED illuminating a single scintillating fiber or the whole PMT (right part of the figure 7).
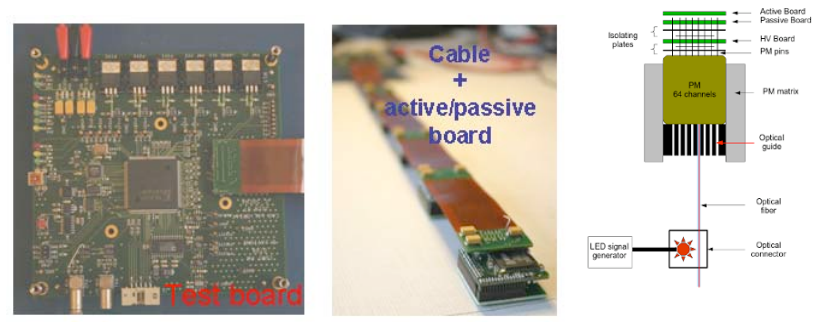

Fig. 7. Left: PMF test board. Center: kapton cable with 5 couples activepassive boards. Right: LED test set-up.

A second lot of 32 pieces was produced end of spring 2008 in order to equip a complete Roman Pot for beam tests during following summer.

\section{B. Prototypes}

\section{1) Standard electrical tests}

All the main characteristics were measured for five active boards out of 8: pedestals, DAC linearity, S-curves (trigger efficiency as a function of the injected charge or the threshold), cross talk and charge.

All of them were found in good agreement with the results obtained during the characterisation of MAROC2 with particularly a nice homogeneity of the pedestals and scurves.

\section{2) Tests with a LED}

Trigger measurements were performed illuminating one or all channels of the MAPMT with a LED for different settings. The parameters tuned were the threshold, the diode pulse amplitude (Vdiode) and the high voltage (HV). 

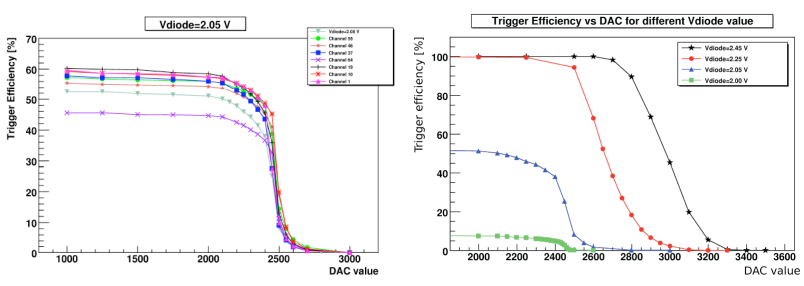

Fig. 8. Left: S-curves of 8 channels for a fixed Vdiode value. Right: Scurves of a single channel for four different Vdiode values.

Left part of figure 8 represents the trigger efficiency, for fixed Vdiode and HV, as a function of the threshold applied to the PMF for 8 different channels. As expected the so called s-curves are observed with a trigger efficiency going from a constant (plateau) value to 0 .

Increasing the amplitude of the LED increases the level of this plateau as it can be seen on the right side of the figure 8 .

A test of the gain equalization was tried by illuminating all channels as uniformly as possible. Figure 9 shows the trigger efficiency as a function of the channel number before and after gain equalization. Dispersion was improved by a factor 3.5 from $13.3 \%$ to $3.8 \%$.

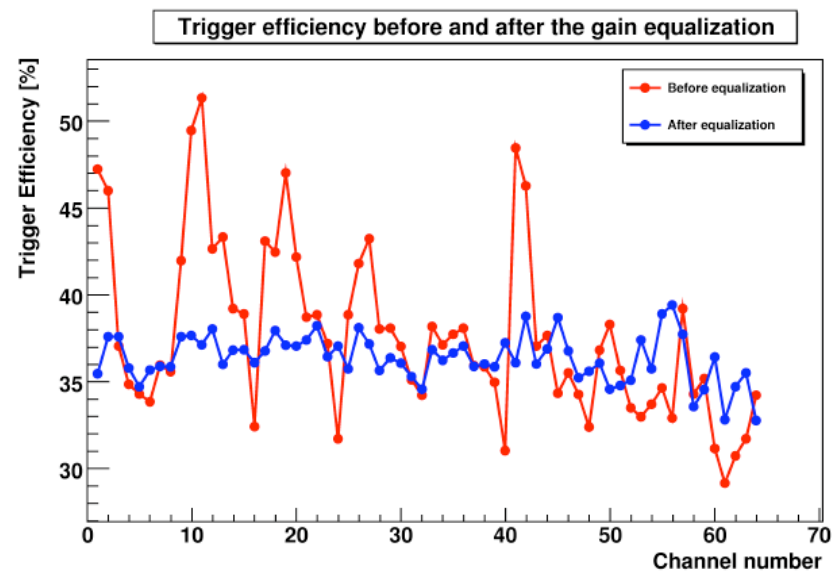

Fig. 9. Trigger efficiency as a function of channel number before and after gain equalization.

\section{Pre-series}

Here are presented results of the measurements performed on 28 active boards out of the 32 .

1) Pedestals

Fast shaper pedestals were measured and a good homogeneity was found as it can be seen on figure 10 which represents the distribution of the pedestal for all the channels of the 28 active boards (i.e. 1792 channels in total). The dispersion is small and equal to $0.4 \%$.

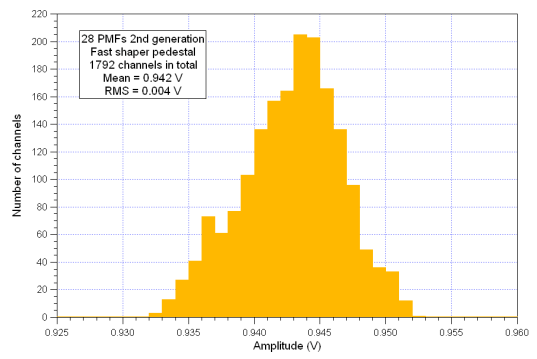

Fig. 10. Fast shaper pedestal distribution for pre-series active boards.

\section{2) DAC linearity}

Similarly the DAC linearity of the 28 active boards has been measured. It consisted in measuring the amplitude (Vdac) obtained for different DAC register values. Top part of figure 11 gives the evolution of Vdac as a function of this register for all boards. The nice homogeneity observed is confirmed by the linear fit (between 0 and 3000) parameters represented as a function of the PMF number (bottom part of figure 10).
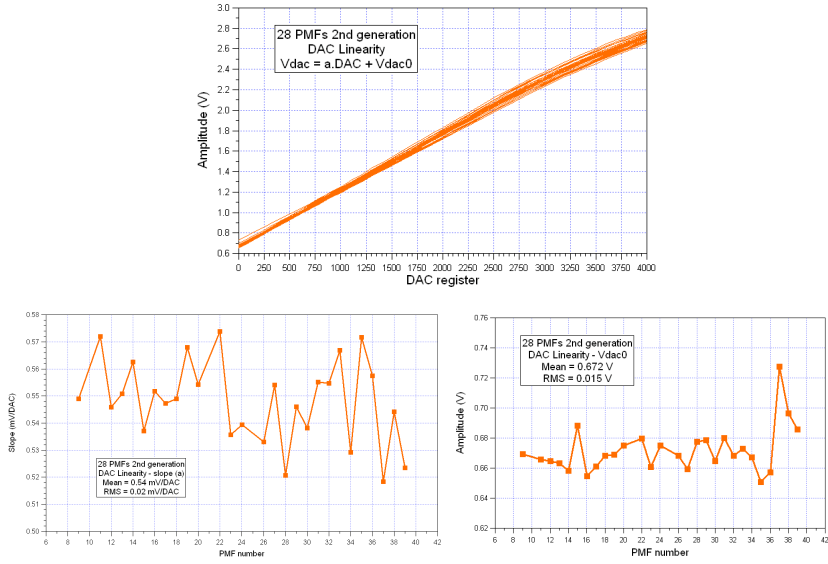

Fig. 11. Top: DAC linearity for the 28 active boards. Bottom left: slope of the DAC linearity fit as a function of PMF number. Bottom right: intercept (Vdac at $\mathrm{DAC}=0$ ) of the DAC linearity fit as a function of PMF number.

\section{3) Trigger outputs}

The trigger output behaviour was studied by scanning the injected charge for a fixed threshold value and a unity gain applied to all channels.

Figure 12 represents the s-curves obtained for 1785 channels out of 1792 . The 7 remaining ones are a bit shifted but nothing that can not be corrected by tuning the gain.

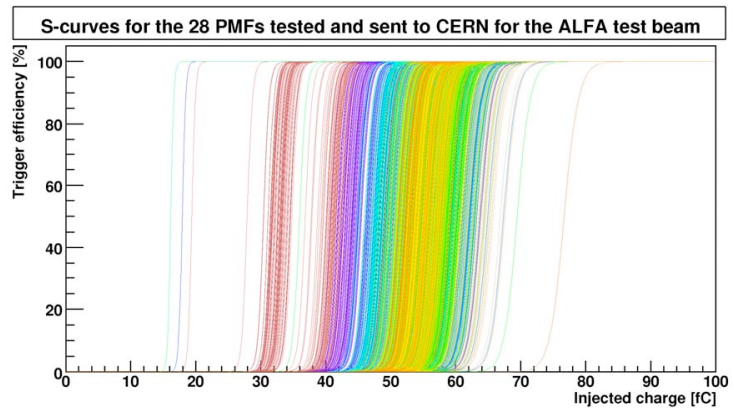

Fig. 12. S-curves for the 28 active boards distinguished by different colors.

The good homogeneity observed can be characterized by the distribution of the injected charge needed to get $50 \%$ trigger efficiency represented on figure 13.

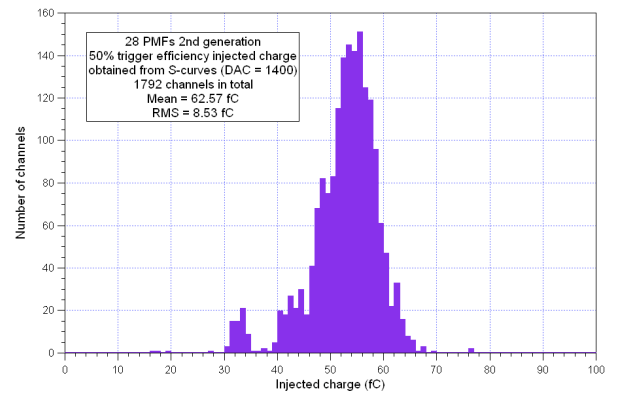

Fig. 13. Distribution of the injected charge needed to get $50 \%$ trigger efficiency among all 1792 channels. 


\section{CONCLUSIONS}

The laboratory tests carried out on prototype and preseries PMFs have showed that they are matching the requirements. The technological challenge consisting in producing a 10 layers PCB with several different vias and an ASIC bonded directly on it seemed to be achieved.

The pre-series batch of active boards showed a really high yield with no board rejected. Only $0.4 \%$ of the channels (7 out of 1792) had an s-curve shifted with respect to the others.

LED tests performed at CERN and DESY proved that the full PMF was also working well.

23 pre-series PMFs were used during beam tests of a complete Roman Pot which took place during August 2008. Results should give the final green light for full production of all three kinds of board.

\section{REFERENCES}

[1] ATLAS Collaboration, ATLAS Forward Detectors for Measurement of Elastic Scattering and Luminosity Determination, Technical Design Report, CERN/LHCC/2008-004.

[2] Hamamatsu web site, PM H7546B datasheet.

[3] G. Blanchot et al., System Design of the ATLAS Absolute Luminosity Monitor, proceedings of TWEPP07 conference.

[4] P. Barrillon et al., 64-channel Front-End readout chip MAROC datasheet.

[5] P. Barrillon et al., MAROC: Multi-Anode ReadOut Chip, proceedings of 2007 IEEE conference. 